\title{
Variedades de Citrus podem afetar a comunidade de fungos do solo?
}

\author{
Kryssia Gislayne Pinheiro Melo*, Angélica Ricarte da Silva, \\ Adriana Mayumi Yano- Melo
}

Universidade Federal do Vale do São Francisco, Petrolina, PE, Brasil

*Autor correspondente, e-mail: kryssiagislayne@hotmail.com

\begin{abstract}
Resumo
Os micro-organismos são imprescindíveis para manutenção da qualidade do solo, dentre os quais se destacam os fungos. Plantas de Citrus podem ser beneficiadas pela associação com fungos micorrízicos arbusculares e presença de fungos solubilizadores de fósforo (FSP) em sua rizosfera, uma vez que estes fungos podem contribuir para o aumento no desenvolvimento das plantas devido a maior disponibilidade de água e nutrientes. Desta forma o presente trabalho teve como objetivo verificar se a quantidade de fungos micorrízicos arbusculares, solubilizadores de $\mathrm{P}$ e totais na rizosfera pode ser afetada por variedades de Citrus. Amostras de solo foram coletadas de um experimento conduzido em delineamento em blocos ao acaso (DBC) com seis variedades de Citrus (Page, Pera D9, Pera D12, Pera C21, Pineaple e Rubi), em quatro repetições. Entre as variáveis quantitativas avaliadas, constatou-se correlação linear simples significativa apenas entre a colonização micorrízica (CM) e o número de unidades formadoras de colônias (UFC/mL) dos FSP, a qual foi negativa. O número de UFC/mL de FSP do solo não diferiu estatisticamente entre as rizosferas das variedades de Citrus, porém para fungos totais (FT) constatou-se diferença significativa, destacando-se a variedade Pineaple que proporcionou maior média no número de UFC/mL, diferindo apenas da variedade Page. Todas as variedades de Citrus apresentaram colonização radicular por FMA e, para o número de glomeroporos (NG) também houve diferença estatística entre as variedades estudadas, porém apenas Rubi e Pineaple diferiram das demais. As variedades de Citrus não afetam a quantidade de FSP e o percentual de CM em suas rizosferas, porém se destacam as variedades Page e Pineaple no aumento do número de glomerosporos de FMA e de UFC/mL de FT.
\end{abstract}

Palavras-chave: fungos micorrízicos arbusculares, fungos solubilizadores de fósforo, fungos totais

\section{Can Citrus varieties affect the soil fungi community?}

\begin{abstract}
Microorganisms are essential for maintaining soil quality, among which stand out the fungi. The association with arbuscular mycorrhizal fungi (AMF) and phosphorous solubilizing fungi (PSF) in its rhizosphere can benefit citrus plants, since these fungi can contribute to an increase in plant development due to the greater availability of water and nutrients. The present study aimed to evaluate if the amount of total fungi, AMF and PSF in the rhizosphere can be affected by the Citrus varieties. Soil samples were collected from the experiment conducted in a randomized block design (RBD) with six Citrus varieties (Page, Pera D9, D12 Pera Pera C21, Pineaple and Ruby). Regarding the evaluated quantitative variables, there was a significant negative simple linear correlation only between the mycorrhizal colonization (MC) and the number of colony forming unit (CFU)/mL of PSF. The number of CFU/mL of soil FPS did not differed statistically between the Citrus varieties rhizosphere, but for total (TF) fungi a significant difference was observed, especially for the 'Pineaple', variety that provided higgher mean for the number of CFU/mL, differing from the 'Page' variety. All Citrus varieties presented root colonization by AMF and there was a significant difference among the evaluated varieties, however only 'Rubi' and 'Pineaple' differed from the others. Citrus varieties do not affect PSF quantity nor MC percentual in their rhizosphere but 'Page' and 'Pineaple' varieties stand out for glomerospores quantity of AMF and TF CFU/mL.
\end{abstract}

Keywords: arbuscular mycorrhizal fungi, phosphorus solubilizing fungi, total fungi. 


\section{Introdução}

A qualidade do solo baseia-se no equilíbrio entre os fatores químicos, físicos e biológicos. No fator biológico diversos processos são mediados por micro-organismos (Moreira et al., 2010) e muitos problemas relacionados à degradação ambiental têm sido mitigados com o seu uso; dentre estes, os fungos representam 10 - 20\% de toda a biomassa microbiana do solo (Hoorman, 2011).

Os Fungos Micorrízicos Arbusculares (FMAs) são amplamente distribuídos nos agroecossistemas, formando simbiose com raízes, cuja interação envolve troca de nutrientes, especialmente C e P (Smith \& Smith, 2012). A simbiose micorrízica propicia maior crescimento e desenvolvimento à planta, aumentando a absorção de água e nutrientes, com consequente aumento na tolerância a fatores bióticos e abióticos (Augé et al., 2015. 2008; Maia et al., 2006).

Estudos demonstram que plantas de Citrus micorrizadas possuem maior crescimento, área foliar, taxa fotossintética e teor de $\mathrm{P}$ foliar, em comparação às plantas não micorrizadas (Smith \& Read, 2008; Wang et al., 2015). Dentre estas, a laranja Poncirus trifoliata apresenta alta dependência micorrízica, com maior biomassa da parte aérea e radicular em planta micorrizada (Wang et al., 2014). Neste sentido, a aplicação de FMA torna-se um fator importante no estabelecimento das plantas em condições de campo (Ortas \& Ustuner, 2014).

Outros grupos de fungos podem ser encontrados na rizosfera de Citrus e proporcionar benefícios, tais como os fungos solubilizadores de $\mathrm{P}$ (FSP), conhecidos por aumentar a disponibilidade de $\mathrm{P}$ devido à liberação de ácidos orgânicos e enzimas ao solo (Singh \& Reddy, 2011). Ademais, trabalhos indicam que o cultivo de Citrus em consórcio com plantas leguminosas pode aumentar a atividade destes fungos (Buzinaro et al., 2009). Neste sentido, a ação combinada de FMA e FSP pode ser favorável à disponibilização de $P$ ao solo e à maior eficiência de absorção deste nutriente pelas plantas. Ressalta-se que em solos com teores elevados de $\mathrm{P}$ pode haver redução significativa da colonização micorrízica (Balzergue et al., 2011).

Outro fator que pode influenciar a sobrevivência dos fungos no solo é a espécie vegetal, embora estudos a este respeito ainda sejam escassos, principalmente comparando-se genótipos da mesma espécie vegetal. Dentre estes, Youpensuk et al. (2009) verificaram que a esporulação e a colonização micorrízica são pouco afetadas pelas espécies ou variedades de Citrus. Salienta-se ainda, que o monocultivo pode reduzir a abundância de propágulos de FMA e que a introdução de espécies vegetais pode alterar a funcionalidade dos fungos (Kivlin \& Hawkes, 2011). O presente trabalho teve como objetivo verificar se a quantidade de fungos micorrízicos arbusculares, solubilizadores de $\mathrm{P}$ e totais na rizosfera pode ser afetada por variedades de Citrus.

\section{Material e Métodos}

Em setembro de 2013 foi realizado - plantio das variedades de Citrus, na área experimental da Universidade Federal do Vale do São Francisco (9¹9'07,91 ' 'S; 40³3'48,59' 'O), caracterizada por clima semiárido (BSh, Koppen), com solo Argissolo amarelo, cujas características químicas e físicas são apresentadas na Tabela 1.

Tabela 1. Características químicas e físicas do solo Argissolo Amarelo (AA) na área de Citrus

\begin{tabular}{|c|c|c|c|c|c|c|c|c|c|c|}
\hline \multirow[t]{2}{*}{ Solo } & $\mathrm{pH}^{*}$ & $\mathrm{P}^{* *}$ & $\mathrm{~K}^{* *}$ & $\mathrm{Ca}$ & $\mathrm{Mg}$ & $\mathrm{Na}^{* *}$ & $\mathrm{Al}$ & \multirow[t]{2}{*}{ Argila } & Areia & \multirow[t]{2}{*}{ Silte } \\
\hline & $\left(\mathrm{H}_{2} \mathrm{O}\right)$ & $\mathrm{mg} / \mathrm{dm}^{3}$ & \multicolumn{5}{|c|}{$\mathrm{cmol}_{\mathrm{c}} / \mathrm{dm}^{3}$} & & $\mathrm{Kg} / \mathrm{Kg}$ & \\
\hline $\mathrm{AA}$ & 6,2 & 207 & 0,74 & 2,1 & 1,4 & 0,11 & 0,00 & 0,095 & 0,880 & 0,025 \\
\hline
\end{tabular}

O espaçamento era de $6 \times 4 \mathrm{~m}$, entre linhas e entre plantas na linha, respectivamente, em covas preparadas nas dimensões de $40 \mathrm{x}$ 40 x $40 \mathrm{~cm}$, aplicando-se $300 \mathrm{~g}$ de superfostato simples e 30 litros de esterco bovino mineralizado, por cova aos 45 dias antes do transplantio. Foi realizada adubação após o transplantio a cada 15 dias, via fertirrigação, com fontes alternadas (nitrocálcio 5,17kg/fertirrigação e uréia 1,36kg/ fertirigação) contendo Nitrocálcio: $12 \%$ (N), 5\% $(\mathrm{P}), 11 \%(\mathrm{~K}), 13,1 \%$ (Ca) e 0,2\% (B); e uréia: $145 \%$ de N). O sistema de irrigação utilizado foi do 
tipo microaspersão, com vazão individual de aproximadamente 50 litros/planta/hora.

O experimento foi conduzido em delineamento em blocos ao acaso com seis variedades de Citrus (Pera D9, Rubi, Pineaple, Pera D12, Page e Pera C21), consorciadas com feijão, em quatro repetições.

Em dezembro de 2014, amostras de solo de cada variedade foram coletadas, retirandose de cada planta amostras de quatro pontos do solo, distantes cerca de $50 \mathrm{~cm}$ entre si, na projeção da copa, com o auxílio de um trado, a uma profundidade de 0 a $20 \mathrm{~cm}$, sendo homogeneizadas e acondicionadas em sacos plásticos, em que parte foi armazenada em isopor com gelo e em seguida mantida em laboratório sob refrigeração para a quantificação dos fungos totais (FT) e solubilizadores de fósforo (FSP); e a outra parte, mantida à temperatura ambiente para a quantificação dos fungos micorrízicos arbusculares (FMA) e do seu percentual de colonização radicular.

Os FT e OS FSP foram isolados pelo método da diluição em série, na qual cada amostra composta de solo foi suspensa em água destilada esterilizada, agitada por 20 minutos e diluída até a concentração de $10^{-4}$, para se fazer a contagem das unidades formadoras de colônia desses micro-organismos por $\mathrm{mL}$ de solução de solo (UFC/mL) (Menezes \& Assis, 2004 modificado). Em seguida, alíquotas de 1,0 mL das suspensões foram semeadas em triplicata em placas de Petri contendo meios de cultura específicos para cada grupo. A quantificação de FT seguiu a metodologia de Martin (1950), enquanto que para OS FSP foi seguida a metodologia de Sylvester-Bradley et al. (1982). O cultivo destes grupos de micro-organismos foi feito, respectivamente, em meio Martin (10 g de dextrose, $5 \mathrm{~g}$ de peptona, $1 \mathrm{~g}$ de $\mathrm{KH}_{2} \mathrm{PO}_{4^{\prime}} \mathrm{0,5} \mathrm{g}$ de $\mathrm{MgSO}_{4} \cdot 7 \mathrm{H}_{2} \mathrm{O}, 0,033 \mathrm{~g}$ de rosa bengala, 500 mg de cloranfenicol, $20 \mathrm{~g}$ de Agar em $1000 \mathrm{~mL}$ de água destilada) e GL (10 g de glicose, $2 \mathrm{~g}$ de extrato de levedura, $16 \mathrm{~g}$ de ágar em $1000 \mathrm{~mL}$ de água destilada, $50 \mathrm{~mL}$ de $\mathrm{K}_{2} \mathrm{HPO}_{4}(10 \%)$ e $50 \mathrm{~mL}$ de $\mathrm{CaCl}_{2}$ (10\%). As placas contendo os meios de cultivo e as amostras diluídas foram incubadas em câmera B.O.D. a $30^{\circ} \mathrm{C}$ por até 7 dias.

Para a quantificação dos fungos micorrízicos arbusculares (FMAs), a partir de 50 g de cada amostra composta de solo, foi feita a extração dos glomerosporos pela técnica de peneiramento úmido (Gerdemann \& Nicolson, 1963) e centrifugação em água e sacarose 40\% (Jenkins, 1964 modificado). Após extração, o número de glomerosporos (NG) foi avaliado com auxílio de estereomicroscópio. Para o percentual de colonização micorrízica (CM), 0,5 $\mathrm{g}$ da biomassa fresca de raiz foram clareadas e coradas com azul de Trypan seguindo a técnica de Dalpé \& Séguin (2013); e a quantificação foi realizada pelo método de interseção dos quadrantes (Giovannetti \& Mosse, 1980).

Os dados de contagem e percentual foram transformados, respectivamente, em $\log (x+1)$ e arcsen $(x / 100)$. Em seguida, foram submetidos à análise de variância e, quando significativas, as médias foram comparadas pelo teste de Tukey a $5 \%$ de probabilidade. Foi realizado o cálculo do coeficiente de correlação linear de Pearson para todas as variáveis estudadas (NG, CM, FT, FSP). Essas análises foram executadas com auxilio do programa estatístico R 3.1.2 (R Core Team, 2014).

\section{Resultados}

Entre as variáveis quantitativas avaliadas, para as seis variedades de laranja em estudo, constatou-se correlação linear simples significativa apenas entre a colonização micorrízica (CM) e o número de unidades formadoras de colônias (UFC/mL) dos FSP, a qual foi negativa (Tabela 2). Por outro lado, não foram observados valores significativos para as demais

Tabela 2. Coeficientes de correlação linear de Pearson para as variáveis colonização micorrízica (CM), número de UFC/mL de fungos solubilizadores de P (FSP), fungos totais (FT) e número de glomerosporos (NG).

\begin{tabular}{cccc}
\hline & FSP & FT & NG \\
\hline CM & $-0,83^{*}$ & $-0,33^{\text {ns }}$ & $0,24^{\text {ns }}$ \\
FSP & & $0,54^{\text {ns }}$ & $-0,18^{\text {ns }}$ \\
FT & & $-0,54^{\text {ns }}$ \\
\hline *. significativo 5\% & &
\end{tabular}

*: significativo a $5 \%$ de probabilidade pelo teste t. ns: não significativo a $5 \%$ de probabilidade pelo teste t. 
correlações.

O número de UFC/mL de FSP do solo não diferiu estatisticamente entre as rizosferas das diferentes variedades de laranjeiras, enquanto que diferença significativa foi observada para $\circ$ grupo de FT (Figura 1). A variedade que proporcionou maior média para os FT foi a Pineaple $\left(56,9 \times 10^{4}\right)$, diferindo apenas da variedade Page $\left(18,1 \times 10^{4}\right)$.

Com relação à colonização micorrízica, todas as raízes avaliadas foram colonizadas por FMA, porém o percentual de colonização micorrízicanão foisignificativamente influenciado pelas variedades, cujos valores médios oscilaram de $13,7 \%$ (Pera D12) a $21,3 \%$ (Pera C21) (Figura 2a). Por outro lado, maior número de glomerosporos foi observado na rizosfera da variedade Page (44), diferindo apenas das variedades Pineaple $(16,0)$ e Rubi $(14,7)$, cujas médias foram as menores observadas (Figura 2b). Porém, não houve correlação significativa entre NG e CM (Tabela 2).

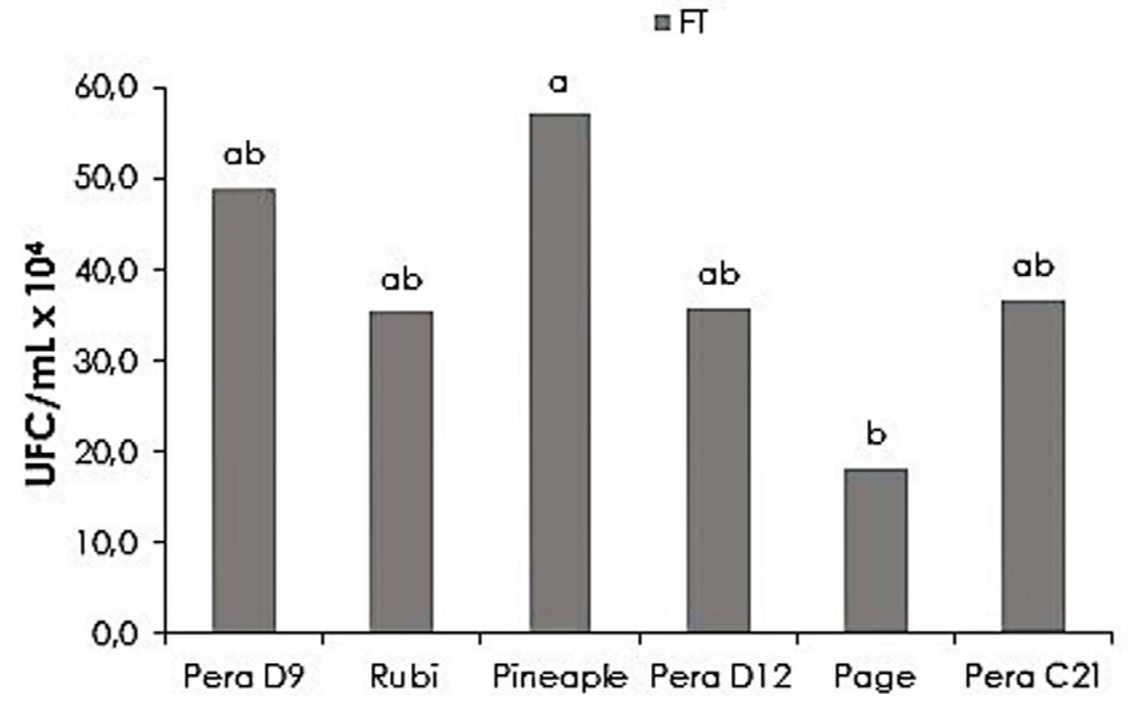

Figura 1. Média do Número de Unidades Formadoras de Colônia (UFC/mL) dos fungos totais (FT) na rizosfera de variedades de Citrus. Letras distintas indicam diferenças significativas entre as variedades, de acordo com teste de Tukey $(P<0,05)$.

a)

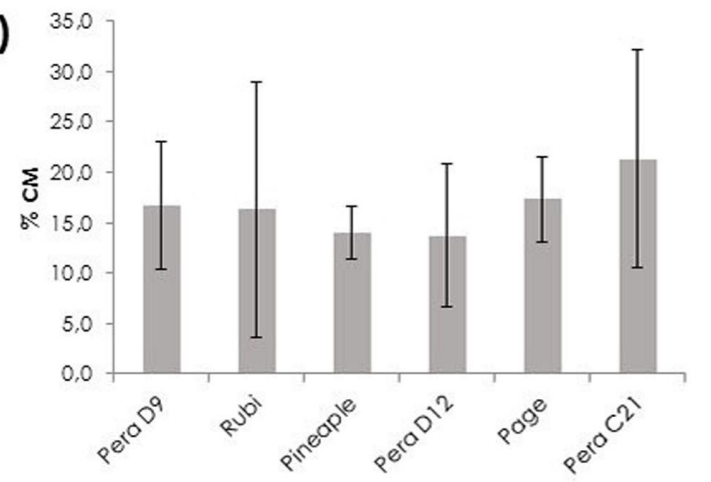

b)

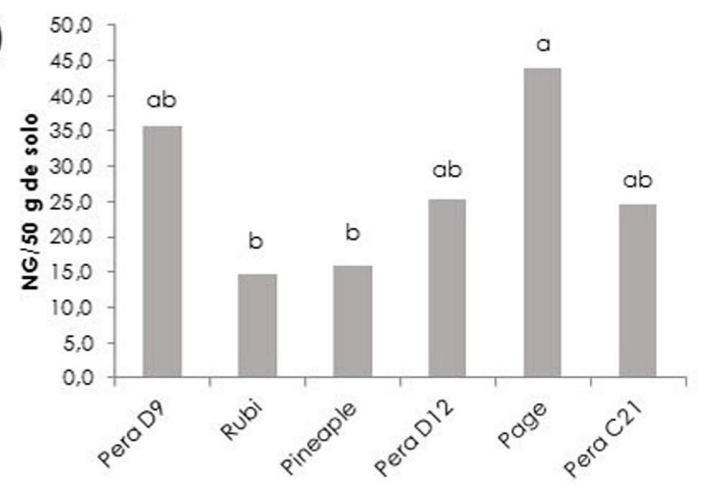

Figura 2. Média da a) Colonização micorrízica (\% CM) e do b) número de glomerosporos na rizosfera de variedades de Citrus. As barras indicam desvio padrão da amostra em cada variedade para CM e as letras distintas indicam diferenças significativas entre as variedades para NG de acordo com teste de Tukey $(\mathrm{P}<0,05)$.

\section{Discussão}

A alta correlação negativa entre a CM e a quantidade de FSP pode ser explicada pela maior disponibilidade de $\mathrm{P}$ na solução do solo por meio da ação desses fungos e consequente redução da necessidade de realização da simbiose entre os FMA e a planta, visto que a capacidade de resposta de plantas micorrizadas à colonização é menor quando cultivadas em solos com fósforo prontamente disponível (Smith \& Smith, 2012). Por outro lado, Della Mónica 
et al. (2014) observaram que plantas de trigo apresentaram maior percentual de colonização micorrízica quando inoculadas com FMA (Gigaspora rosea) em associação com FSP (Talaromyces flavus). Além disso, ainda de acordo com os autores, foi constado efeito significativo da presença de exsudatos de T. flavus sobre o desenvolvimento do micélio pré-infectivo de $G$. rosea. Esta incongruência nos resultados pode indicar que combinações específicas podem favorecer ambos os organismos, sugerindo a seleção de espécies que apresentem sinergismo em sua funcionalidade.

Para a situação do presente estudo, a colonização micorrízica é independente da variedade de laranjeira estudada, tendo em vista que não houve diferença estatística significativa para a CM e o número de UFC/mL dos FSP entre as mesmas. Da mesma forma, Youpensuk etal. (2009) não encontrou diferença estatística significativa entre as variedades estudadas, exceto para a variedade de tangerina Cleópatra, Citrus reshni Hort. ex Tan. Mesmo em diferentes sistemas de produção, que apresentavam características químicas do solo distintas, Focchi et al. (2004) observaram que plantas de Citrus apresentavam colonização por FMA. Tal fato pode sugerir que a dependência micorrízica pode contribuir para a ocorrência de colonização micorrízica encontrada em variedades de citrus. Além disto, Wang et al. (2014)verificaram em experimento em casa de vegetação com P. trifoliata, em solo autoclavado, que a colonização radicular por espécies de FMA pode variar, mas em geral contribuem para a melhoria das propriedades físicas do solo e desenvolvimento das plantas. Os resultados indicam que 0 percentual de colonização micorrízica pode variar em decorrência da espécie de FMA inoculada, porém, em condições de campo a diversidade de espécies de FMA presente pode não resultar em diferença nesta variável. Ausência de diferença na colonização micorrízica entre porta-enxertos de citros, em condições de campo, foram reportadas por Nunes et al. (2006), que encontraram percentuais médios variando de 56 a $69 \%$, mesmo em solos com teores altos de $P\left(100 \mathrm{mg} / \mathrm{dm}^{3}\right)$. Salienta-se que apenas para poucas espécies vegetais os processos que levam à colonização da raiz e a maneira pela qual os FMA se desenvolvem nos sistemas radiculares são bem compreendidos (Smith \& Read, 2008), sugerindo a necessidade de mais estudos sobre este processo.

Os valores de CM observados (13,7 $21,3 \%)$ no campo foram muito inferiores aos encontrados por Nunes et al. (2006) e Wang et al. (2013), cujos valores oscilaram de 50 a $77 \%$ em porta-enxertos de citros. Provavelmente 0 alto teor de $\mathrm{P}\left(207 \mathrm{mg} / \mathrm{dm}^{3}\right)$ na área estudada (Tabela 1) pode ter contribuído para reduzir a colonização micorrízica, visto que nas áreas avaliadas por Nunes et al. (2006) e Wang et al. (2013) os valores não excediam $100 \mathrm{mg} / \mathrm{dm}^{3}$. Em casa de vegetação, Wang et al. (2014) também encontraram valores superiores, que variaram de $32,1 \pm 3,5 \%$ a $59,1 \pm 4,8 \%$ em plantas de Poncirus trifoliata L. Raf. inoculadas com cinco espécies de FMA (Diversispora spurca, Glomus intraradices, Glomus mosseae, Glomus versiforme, e Paraglomus occultum), indicando que dependendo da espécie de FMA inoculada os percentuais de colonização micorrízica podem ser significativamente afetados. Valores entre 75 e $96 \%$ de colonização micorrízica foram encontrados por Youpensuk et al. (2009) ao trabalharem com diferentes variedades de Citrus, estes percentuais foram alcançados inoculando-se uma mistura de 20 espécies de FMA, aumentando consideravelmente a possibilidade de colonização radicular. Neste sentido, constata-se que a ampla variação nos percentuais de colonização micorrízica podem ser influenciados por diferentes fatores, tais como fertilidade do solo, especialmente P, e espécies de FMA no solo.

Embora Singh et al. (2012) ressaltem que o genótipo pode ser uma fonte significativa de variação nos valores de CM, sob condições de baixa e média fertilidade do solo, de forma a mostrar existência de variação genética na compatibilidade de uma variedade com FMA, estes aspectos nem sempre são claramente observados, a exemplo das variedades de Citrus avaliadas em nosso estudo. Singh et al. (2012) afirmam também que um genótipo responsivo à micorrização pode mostrar baixa performance em comparação a outros genótipos da mesma 
espécie. Desta forma, estudos de responsividade à micorrização das variedades de Citrus avaliadas podem auxiliar na compreensão e melhor manejo das comunidades de FMA no solo.

Também não foi observada correlação significativa entre a CM e $\circ$ NG. Embora a determinação do número de esporos no solo possa apresentar relação com a raiz colonizada, nem sempre isso acontece, tendo em vista que apesar dos esporos estarem disponíveis como inóculo para formação da colonização, esse processo dependerá muitas vezes das condições do solo (Youpensuk et al., 2006; Smith \& Read, 2008). Além destes aspectos, a ausência de correlação entre estas variáveis pode ser decorrente da composição da comunidade de FMA na rizosfera de cada variedade de Citrus. Corrobora para esta afirmação o estudo de Weber \& Oliveira (1994), que observaram maior número de esporos e intensidade de colonização micorrízica nas raízes, além de grande variação da comunidade de FMA em solos de baixa fertilidade.

Observou-se variação no NG de 2,9 a 8,8 em $10 \mathrm{~g}$ de solo para as variedades estudadas, fato também constatado por Youpensuk et al. (2009) ao avaliarem variedades de tangerina e espécies cítricas comuns, cuja variação foi de 14 a 28 glomerosporos por $10 \mathrm{~g}$ de solo. Embora o número de glomerosporos na rizosfera das variedades de Citrus no presente estudo tenha sido baixo, estes valores estão na faixa encontrada em áreas da Caatinga (Maia et al., 2010).

Foi observada diferença estatística significativa para o número de UFC/mL dos fungos totais apenas entre duas variedades (Pineaple e Page), as quais não diferiram das demais. Isso sugere que não haja variações extremas sobre a quantidade desses fungos no solo em virtude da utilização de diferentes variedades para a mesma espécie de Citrus. Porém, quando se comparou diferentes espécies vegetais (Olea europeae, Citrus sinensis e Eucalyptus rostrata) como fontes de matéria orgânica para fungos do solo, Abdel-Hafez \& Abdel-Fattah (1981) constataram que folhas de C. sinensis aumentam significativamente a população fúngica, considerando-a uma fonte de substrato mais adequada para promover o aumento da população. Em nosso estudo, a variedade Pineaple foi mais favorável ao incremento do número de fungos totais do solo, podendo sugerir maior potencial para promover a funcionalidade dos fungos decompositores.

Para Wang et al. (2013), os fatores ambientais são importantes na determinação da colonização micorrízica, do número de propágulos e da diversidade espécies de FMA em pomares de Citrus. Neste sentido, a identificação morfológica ou molecular das espécies de FMA na rizosfera de Citrus podem indicar mais claramente a influência destes genótipos sobre a propagação dos FMA e desta forma planejar a adoção de manejos que favoreçam as populações fúngicas benéficas.

\section{Conclusões}

As diferentes variedades de Citrus não influenciam o número de Unidades Formadoras de Colônia (UFC/mL) de fungos solubilizadores de fósforo do solo e a colonização micorrízica, salientando que todas as variedades formam associação com fungos micorrízicos arbusculares (FMA). Nas condições experimentais do presente estudo, destacam-se as variedades Page por proporcionar o maior número de glomerosporos e a Pineaple com maior número de UFC/mL dos fungos totais.

\section{Agradecimentos}

Ao professor ítalo Herbert Lucena Cavalcante pelo apoio para realização do experimento, à Facepe, ao CNPq e à Univasf.

\section{Referências}

Abdel-Hafez, S.I.I., Abdel-Fattah, H.M. 1981. Effect of carbon levels from three organic substrates on egyptian soil fungi. Plant and Soil 60: 65-72.

Augé, R.M., Toler, H.D., Saxton, A.M. 2015. Arbuscular mycorrhizal symbiosis alters stomatal conductance of host plants more under drought than under amply watered conditions: a metaanalysis. Mycorrhiza 25: 13-24.

Balzergue, C., Puech-Pagès, V., Bécard, G., Rochange, S.F. 2011 . The regulation of arbuscular mycorrhizal symbiosis by phosphate in pea involves early and systemic signaling events. Journal of Experimental Botany 62 (3): 1049-1060. 
Buzinaro, T.C., Barbosa, J.C., Nahas, E. 2009. Atividade microbiana do solo em pomar de laranja em resposta ao cultivo de adubos verdes. Revista Brasileira de Fruticultura 31 (2): 408-415.

Dalpé, Y., Séguin, S.M. 2013. Microwave-assisted technology for the clearing and staining of arbuscular mycorrhizal fungi in roots. Mycorrhiza 23: 333-340.

Della Mónica, I.F., Stefanoni Rubio, P.J., CINA, R.P., Recchi, M., Godeas, A.M., Scervino, J.M. 2014. Effects of the phosphate-solubilizing fungus Talaromyces flavus on the development and efficiency of the Gigaspora rosea-Triticum aestivum symbiosis. Symbiosis 64: 25-32.

Focchi, S.S., Soglio, F.K.D., Carrenho R., Souza, P.V.D., Lovato, P.E. 2004. Fungos micorrízicos arbusculares em cultivos de citros sob manejo convencional e orgânico. Pesquisa Agropecuária Brasileira 39(5): 469-476.

Gerdemann, J.W., Nicolson, T.H. 1963. Spores of mycorrhizal Endogone species extracted from soil by wet sieving and decanting. Transactions of the British Mycological Society 46: 235-244.

Giovannetti, M., Mosse, B. 1980. An evaluation of techniques to measure vesicular-arbuscular mycorrhizal infection in roots. New Phytologist 84: 484-500.

Hoorman, J.J. 2011. The Role of Soil Fungus. The Ohio State University Extension, Columbus, EUA. 6 p. (Agriculture and Natural Resources SAG-14-11)

Jenkins, W.R. 1964. A rapid centrifugal-flotation technique for separating nematodes from soil. Plant Disease Report 48: 692.

Kivlin, S.N., Hawkes, C.V. 2011. Differentiating between effects of invasion and diversity: impacts of aboveground plant communities on belowground fungal communities. New Phytologist 189: 526-535.

Maia, L.C., Silva, G.A., Yano-Melo, A.M., Goto, B.T. 2010. Fungos micorrízicos arbusculares no bioma Caatinga. In: Siqueira, J.O., Souza, F.A., Cardoso, E.J.B.N., Tsai, S.M. (Ed.). Micorrizas: 30 anos de pesquisas no Brasil. Ed. UFLA, Lavras. p. 31 1-339.

Maia, L.C., Silveira, N.S.S., Cavalcante, U.M.T. 2006. Interaction between arbuscular mycorrhizal fungi and root pathogens. In: Rai, M.K. (Ed.). Handbook of microbial biofertilizers. The Haworth Press Inc., New York. p. 325-352.

Martin, J.P. 1950. Use of acid, rose Bengal and streptomycin in the plate method for stimating soil fungi. Soil Science 69: 215-232.

Menezes, M., Assis, S.M.P. 2004. Guia prático para fungos fitopatogênicos. 2 ed. Recife: UFRPE,
$183 p$.

Moreira, F.M.S., Silva, K., Nóbrega, R.S.A., Carvalho, F. 2010. Bactérias diazotróficas associativas: diversidade, ecologia e potencial de aplicações. Comunicata Scientiae 1(2): 7499

Nunes, M.S.; Soares, A.C.F.; Soares Filho, W.S.; Lêdo, C.A.S. 2006. Colonização micorrízica natural de porta-enxertos de citros em campo. Pesquisa Agropecuária Brasileira 41 (3): 525-528.

Ortas I., Ustuner O. 2014. The effects of single species, dual species and indigenous mycorrhiza inoculation on citrus growth and nutrient uptake. European Journal of Soil Biology 63: 64-69.

R Core Team 2014. R: A language and environment for statistical computing. $\mathrm{R}$ Foundation for Statistical computing. http://www.R-project. org/<Acesso em novembro de 2014>

Singh, A.K., Hamel, C., Depauw, R.M., Knox, R.E. 2012. Genetic variability in arbuscular mycorrhizal fungi compatibility supports the selection of durum wheat genotypes for enhancing soil ecological services and cropping systems in Canada. Canadian Journal of Microbiology58: 293-302.

Singh, H.; Reddy, M.S. 2011. Effect of inoculation with phosphate solubilizing fungus on growth and nutriente uptake of wheat and maize plants fertilized with rock phosphate in alkaline soils. European Journal of Soil Biology 47: 30-34.

Smith, S.E., Read, D.J. 2008. Mycorrhizal Symbiosis. 3 ed. San Diego: Academic Press, Amsterdam, The Netherlands. $86 \mathrm{p}$.

Smith, S.E., Smith, F.A. 2012. Fresh perspectives on the roles of arbuscular mycorrhizal fungi in plant nutrition and growth. Mycologia 104 (1): 1-13.

Sylvester-Bradley, R., Asakawa, N., La Torraca, S., Magalhães, F.M.M., Oliveira, L. A., Pereira, R.M. 1982. Levantamento quantitativo de microrganismos solubilizadores de fosfatos na rizosfera de gramíneas e leguminosas forrageiras na Amazônia. Acta Amazônica 12: 15-22.

Wang, P., Wang, Y., Shu, B., Liu, J., Xia, R.X. 2015. Relationships between arbuscular mycorrhizal symbiosis and soil fertility factors in Citrus orchards along an altitudinal gradient. Pedosphere 25 (1): 160-168.

Wang, P., Wang, Y., Zhang, D.J., Liu, J.F., Xia, R.X. 2013. Diversity of arbuscular mycorrhizal fungi in red tangerine (Citrus reticulate Blanco) rootstock rhizospheric soils from hillside citrus orchards. Pedobiologia 56: 161- 167.

Wang, S., Srivastava, A.K., WU, Q-S., Fokom, R. 
2014. The effect of mycorrhizal inoculation on the rhizosphere properties of trifoliate orange (Poncirus trifoliata L. Raf.). Scientia Horticulturae 170: 137-142.

Weber, O.B., Oliveira, E. 1994. Ocorrência de fungos micorrízicos vesículo-arbusculares em citros nos estados da Bahia e Sergipe. Pesquisa Agropecuária Brasileira 29(12): 1905-1914.

Youpensuk, S., Lordkaew, S., Rerkasem, B. 2006.

Comparing the effect of arbuscular mycorrhizal fungi on upland rice and macaranga denticulata in soil with different levels of acidity. Science Asia 32: 121-126.

Youpensuk, S., Lordkaew, S., Rerkasem, B. 2009. Genotypic variation in responses of Citrus spp. to arbuscular mycorrhizal fungi. Journal of Agricultural Science 1 (1): 59-65. 